\title{
Thermal and Electrochemical Properties of Polymannuronate-polyaniline Nanocomposites
}

\author{
C. Basavaraja, Y. Veeranagouda, ${ }^{+}$Na Ri Kim, Eun Ae Jo, Kyoung Lee, and Do Sung Huhr \\ Department of Chemistry and Institute of Functional Materials, Inje University, Kimhae, Kiungnam 621-7+9, Korea \\ E-mail: chemhdsainje ack $k$. \\ ${ }^{\dagger}$ Department of Aficrobiology, Changwion Enversity, Changwon, Kyongnam 641-773, Korea \\ Received February 27, 2009. Accepted March 23, 2009
}

\begin{abstract}
New types of conducting polyaniline-polymannuronate (PANI-PM) composites were synthesized by in sith deposition techniques in an aqueous media. By dissolving different weight percentage of polymannuronate (PM) $(5$, 10,15 , and $25 \%$ ), the oxidative polymerization of aniline was carried out using ammonium per sulfate as an oxidant. The obtained composites were studied for their thermal stability and electrochemical behavior. The thermal stability of PANI-PM composites is lower than PANI, which supports a strong interaction between PANI and PM. However, the composites show an appreciable electrochemical behavior. Based on these observation the PANI-PM composites can be explored in different fields such as electric devices, sensors, functional coatings, efc.
\end{abstract}

Key Wonds: Conducting polymer. Polyaniline. Polỵmannuronate. Nanocomposites. Themal stabiliț

\section{Introduction}

The development of intrinsically conducting polymers (ICP) has attracted great attention by the scientific community owing to the increased potential for technological applications in electrochromic devices, sensors, electrolytic capacitors. rechargeable batteries. etc. ${ }^{i}$ Among the polymers. polyaniline (PANI) generates a special interest owing to its relatively low cost. physico-chemical stability. and a good combination of optical. catalytic. conductive, and sensor properties. ${ }^{2.3}$ However, a major problem still exists in the practical use of PANI and other ICP because of their infusibility. insolubility to an aqueous solution, and poor mechanical characteristics. An alternative approach is the use of PANI in conducting composites or blends with conventional poly mers. ${ }^{4,5}$ The obtained composites combine good conductive properties with mechanical and even optical properties of the polymer matrix. This allows the development of polymeric composites with specific properties and shapes. ${ }^{6-10}$ For synthesizing such composite material, various attempts have been made to blend PANI with insulating polymers that have good mechanical strength. ${ }^{1: i-13}$ Many effective efforts have been directed toward enhancing the electroactivity of PANI ${ }^{11.13}$ either by introducing acidic groups into the PANI chains ${ }^{14}$ or doping PANI with negatively charged polyelectrolytes. ${ }^{15}$ Recent advances in the development of fabrication technology to the micro or nano scale to obtain an inexpensive signal processing systems have made a variety of novel biomedical sensors possible. ${ }^{1(11.11}$ Thus it is a very demanding process to synthesize new PANI containing appropriate material suitable for improved electrical and processable properties better than PANI. The introduction of biopolymers especially in the polymer matrix of PANI makes the process of synthesis easier because of the high solubility of biopolymers either partially or completely in aqueous solvents. ${ }^{16}$

Polymannuronate (PM) is a good biopolymer for the purpose since it has a well-defined chemical composition; it has therefore attracted special interest in bionedical research. PM is a component of alginate and has been shown to possess many medical and pharnaceutical applications. ${ }^{1 ? 18}$ The administration of PM has been shown to protect mice from lethal X-ray irradiation and also stimulates the generation of murine myeloid progenitor system. ${ }^{19}$ In vivo animal models have now revealed the immunologic potential of PM in such diverse areas as the protection against lethal bacterial infections and irradiation. and increasing non-specific immunity. ${ }^{\text {ji }}$ In this report. PANI-PM conducting composites have been synthesized with different weight percentages of PM (5, 10 . 15 , and $25 \%$ ). The PANI-PM conducting composites were studied for their thernal behavior by themogravimetric (TGA), and the electrochemical properties of these composites were studied by cyclic voltammetric measurements.

\section{Experimental}

Materials. All the chemicals used for the synthesis of PANI and its composites were purchased from Sigma-Aldrich and used without further purification.

Production of PM from bacteria. The production of PM from the bacterial strain $(\mathrm{E} 1)$ is derived from Pseudomonas $\mathrm{sp}$ $\mathrm{KL} 28$. which was previously isolated as n-alkyl phenol degrading bacterium. ${ }^{21}$ The modified King's medium (PG) used for the production of PM consists of $(\mathrm{g} / \mathrm{L})$ peptone $(20)$. glycerol (10), and $\mathrm{MgSO}_{4}(1.5)$. The medium was prepared by phosphate buffer of $50 \mathrm{mM}$ with $\mathrm{pH} 6.0$ sterilized by autoclaving at $15 \mathrm{lb}$ for $20 \mathrm{~min}$. Inoculum was prepared by growing a single colony in $5 \mathrm{~mL} \mathrm{LB}$ medium for $10 \cdot 12 \mathrm{~h}$ at $30^{\circ} \mathrm{C}$. The $50 \mu \mathrm{L}$ of the inoculum was used to inoculate a 250 $\mathrm{mL}$ Erlenmeyer flask containing $50 \mathrm{~mL}$ of medium. After then. the flasks were incubated at $30^{\circ} \mathrm{C}$ on a rotary shaker at $160 \mathrm{rpm}$ for 3 days. The PM in culture supernatant was precipitated by the addition of an equal volume of isopropyl alcohol and the precipitate was removed by a glass rod. The precipitate was redissolved in water and reprecipitated with 


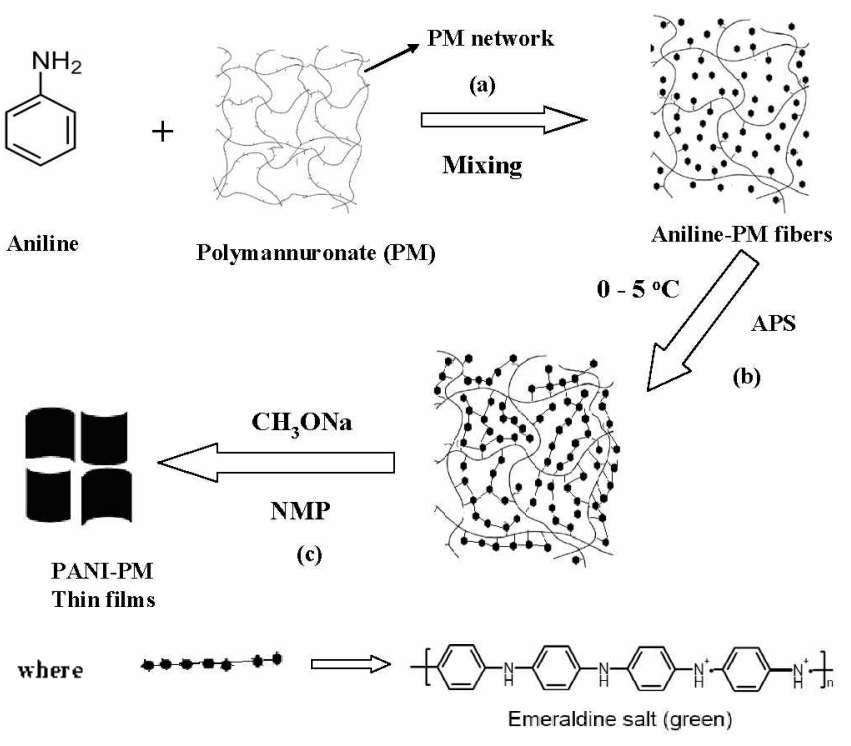

Figure 1. Polymerization of PANI in the presence of PM microgels to form the PANI-PM composite. (a) Incorporation of Aniline into the PM network (b) Polymerization of aniline-PM to form the PANI-PM composite (c) Fornation of PANI-PM thin films.

three volumes of ethanol. The resulting PM was washed several times with $100 \%$ ethanol and dried at $80^{\circ} \mathrm{C}$ to obtain constant weight. It was then milled using a pestle and mortar and used for composite syththesis

Synthesis of PANI and PANI-PM composites. A weighed amount of PM powder was dissolved in $200 \mathrm{~mL}$ distilled water. and then $2.0 \mathrm{~mL}$ of aniline was injected into this suspension. which was stirred magnetically for $2 \mathrm{~h}$. Thereafter $0.1 \mathrm{M}$ of APS was slowly added to the mixture with constant stirring and the reaction was carried out for about $4.5 \mathrm{~h}$ at 0 ${ }^{\circ} \mathrm{C}$. The precipitated composite was filtered and washed several times with acetone and distilled water. The target mass loading of monomer in the composites was from 5 to $25 \mathrm{wt} \%$. PANI homopolymer was sy nthesized under the same conditions for comparison with the composites. The composites are abbreviated as PANI $-v$, where $y$ refers to the $w t \%$ of PM powder used in the polymerization reaction. After mixing aniline with the solution containing PM which shows a slightly' brown-colored solution. the aniline-PM complex began to form during mixing. Following the addition of APS to this reaction mixture, the PANI-PM composite was formed

Figure 1 depicts a schematic model for the formation of the PANI-PM composite structure. The figure illustrates how the microgels structure of PM stabilizes the dispersion of PANI. The fibrillar structure of PM adsorbs the aniline to form the aniline-PM complex after aniline polymerization in order to obtain the PANI-PM composite. withholding the chain of PANI into the PM matrix. It indicates that the microgel structures of PM stabilizes the dispersion of PANI: this modifies into a uniform surface withholding PANI particles in the fibers formed by PM in the form of network as indicated in the figure. In our earlier report a detailed synthesis and characterization of the PANI-PM composites has been explained. The interaction between PANI and PM are studied using different characterization techniques. ${ }^{2 . .2 \hat{3}}$ It has been suggested that the interaction between PANI and PM increases in the composite. This may be due to the molecular chain of PM that contain polymannuronic acid units which are flat and forms ribbon-like structures. These structures are further stabilized by intramolecular hydrogen bonding. And also PM has free hydrosyl groups and negatively charged carbonyl groups and may help to increase the interaction after doping PM with PANI.

Measurements. In this study the thermal properties of the sy nthesized PANI and PANI-PM composites were studied by TGA (Perkin Elmer model TGA 7) in the range of $25-800^{\circ} \mathrm{C}$ at $10^{\circ} \mathrm{C} / \mathrm{min}$ in nitrogen atmosphere. Electrochemical measurements were carried out using the multifunctional analyzer Model 660 of $\mathrm{CH}$ Instruments (Cordova. TN. U.S.A.). All experiments were carried out using a conventional threeelectrode systent with the enzyme platinum electrode as the working electrode and an $\mathrm{Ag} / \mathrm{AgCl}$ (saturated $\mathrm{KCl}$ ) electrode as the reference electrode. Electrolyte solutions were purged with high-purity nitrogen prior to. and blanked with nitrogen during, electrochenical experiments. Electrochenical experiments were carried out in $0.5 \mathrm{M} \mathrm{H}_{2} \mathrm{SO}_{4}$. The current response of the electrode was measured between -0.2 and $1.2 \mathrm{~V}$ at the different scan rates from 10 to $60 \mathrm{mV} \mathrm{s}^{\cdot .}$.

\section{Results and Discussion}

Themal properties of PANI and PANI-PM composites. Figure 2 shows the thermogravimetric analysis (TGA) of PANI, PANI-15, and PANI-25 composites. The composites undergo three stages of degradation, though the third stage is not visible clearly in the case of PANI-25. The initial weight loss in the range of $50-130^{\circ} \mathrm{C}$ is due to the loss of moisture. and the second stage begins after $230^{\circ} \mathrm{C}$ as the polymer is thermally stable unto this temperature: thereafter the third stage proceeds up to $450-500^{\circ} \mathrm{C}$ from where a rapid degradation begins. The maxinum degradation temperatures for these composites are around $660^{\circ} \mathrm{C}$. The TGA curves of PANI-15 and PANI-25 show the loss of moisture in the first step of decomposition same as PANI and the removal of dopant anions and decomposition of the materials in the subsequent steps. The initial decomposition temperatures of PANI-15 and PANI-25 composites in the second and third steps are lower than for PANI. ${ }^{24}$ This shows that PANI-15 and PANI-25 are less stable than PANI. Compared to PANI-15 and PANI-25. PANI-25 is more stable similar to PANI owing to the aggregation of polymer matrix, which can be evidenced by SEM images showing a decrease in the particle size of the composite.

Cyclic-voltammetry measurements of PANI and PANI-PM composites. Figures $3(\mathrm{a})$ and $3(\mathrm{~b})$ show cyclic voltammogranis of PANI-15 and PANI-25, respectively, at the scan rates of $10-60 \mathrm{mV} \mathrm{s}^{-1}$. The scan rate dependency in PANI-15 and PANI-25 shows that the redox transformations are fast and the mass changes are accompanied by the redox reactions. which decrease with an increase in the scan rate. Since the peak currents are proportional to the scan rate. all the waves behave like the surface waves at low scan rates. This may be due to the diffusion behavior at the surface. The composite samples measured by varying scan rates showed that the peak currents increase linearly with the scan rates indicating a surface- 


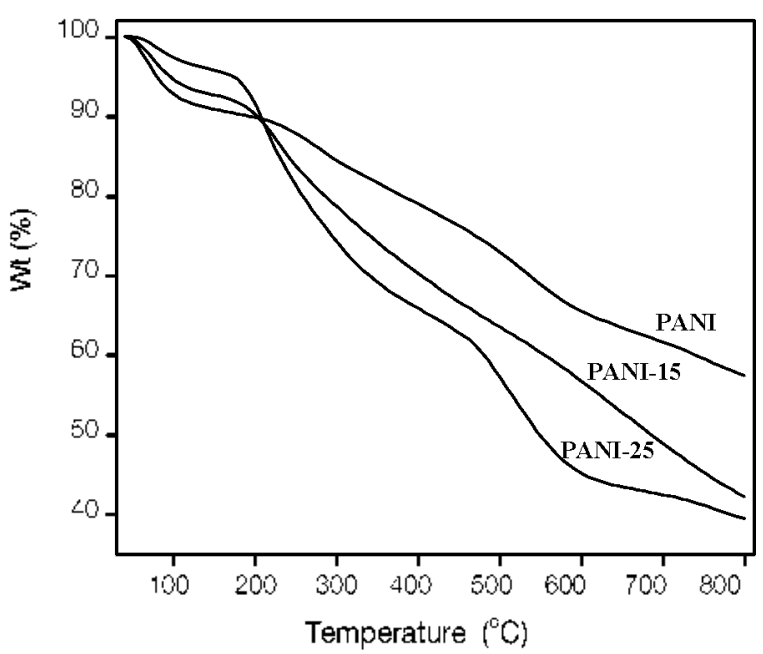

Figure 2. TGA of PANI, PANI-15, and PANI-25 composites.

controlled redox process, which in tum indicates a diffusioncontrolled mechanism.

Figure 4 shows the voltammograms of PANI. PANI-15, and PANI-25 at the scan rate $10 \mathrm{mV} \mathrm{s}^{.1}$. The linear dependence of the peak currents on scan rates indicates that the redox process is fast. The electrochemical behavior of PANI-PM composites may originate from the fact that both PANI and PM are conducting. which facilitates a fast charge transfer across the solution. With an increase in the scan rate for PANI-15 and PANI-25, the anodic and cathodic peak potentials shift towards more positive and negative direction, respectively. as shown in Figures 3(a) and 3(b) and this behavior is similar to that of PANI/carbon nanotube multilayer films prepared via the lay'er-by-lay'er method. ${ }^{25}$ Through close comparisons of the CV curves between PANI- 15 and PANI-25 and pure PANI in Figure $t_{\text {, }}$ it is observed that not only does PANI-PM display a higher background current in the potential sweep but also there exist faradaic currents, which are believed to arise from the contribution of the loaded PM. It has been reported that PANI was considered as a promising material for electrochemical capacitors because of the existence of different oxidation states. Owing to the higher current in the voltammograms of the PANI-PM electrode than that in a pure PANI. ${ }^{26}$ a larger capacitance for capacitors equipped with PANI-PM electrode can be anticipated

The molecular dispersion of PM in solution forms a microgel state. which makes the particles a continuous electron path that generates more active sites for the charge transfer through the interface inside the electrode by making good contacts with the PANI matrix. In the cyclic voltammetric curves, the PANI-PM composite core-shell shows appreciable redox activity. The molecular chain of PM is flat and ribbon-like. whose conformation is stabilized by the formation of an intramolecular hydrogen bond in the solution. By the result. the PANI-PM composite macromolecules show very rigid and extended patterns. This makes a continuous electron path by charge transfer at the interface between the electrodes. Thus PANI particles located far from the electrode surface can effectively take part in the redox reaction. ${ }^{28}$ The electrochemical activity of these composites is enhanced by a slight
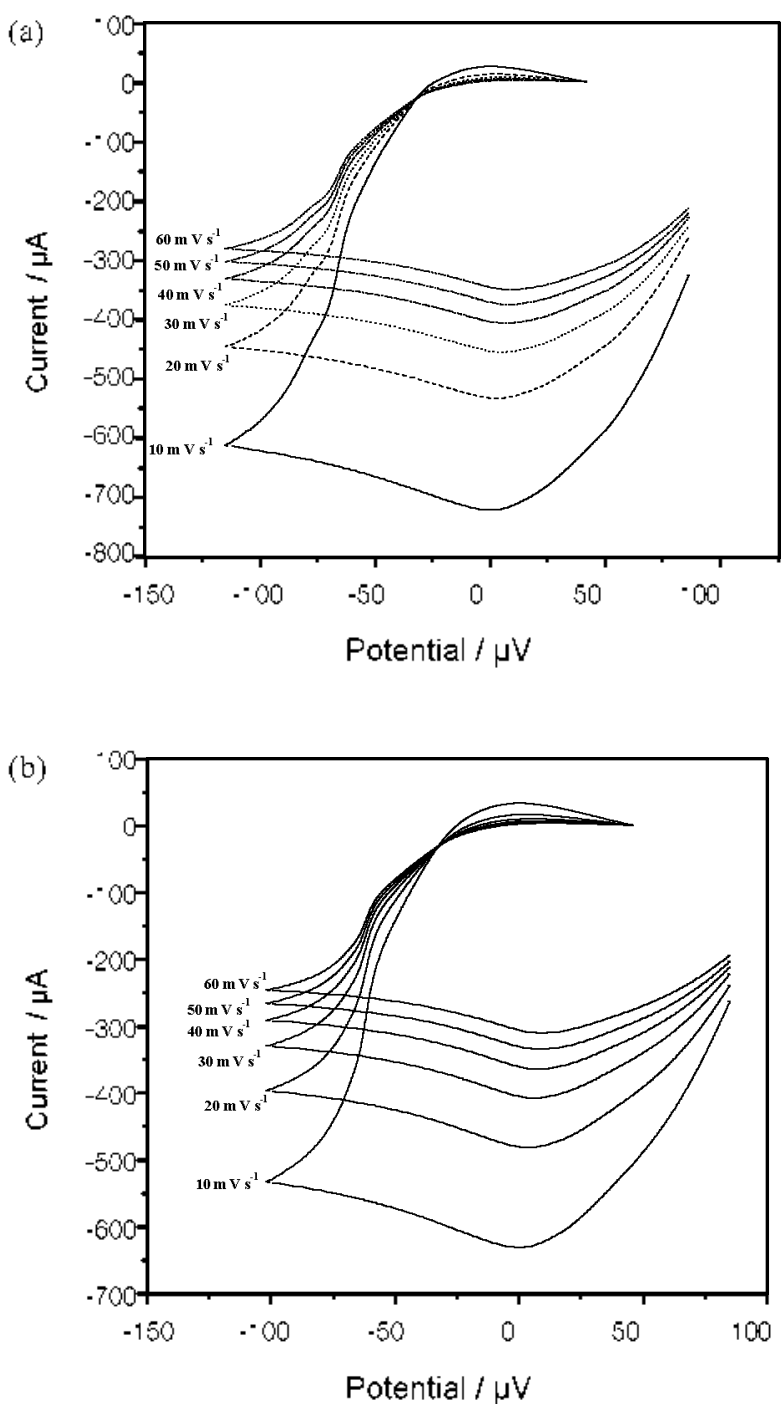

Figure 3. Cyclic voltammograms for (a) PANI-15 and (b) PANI-25 at scan rates of $10-60 \mathrm{mV} \mathrm{s}^{-1}$

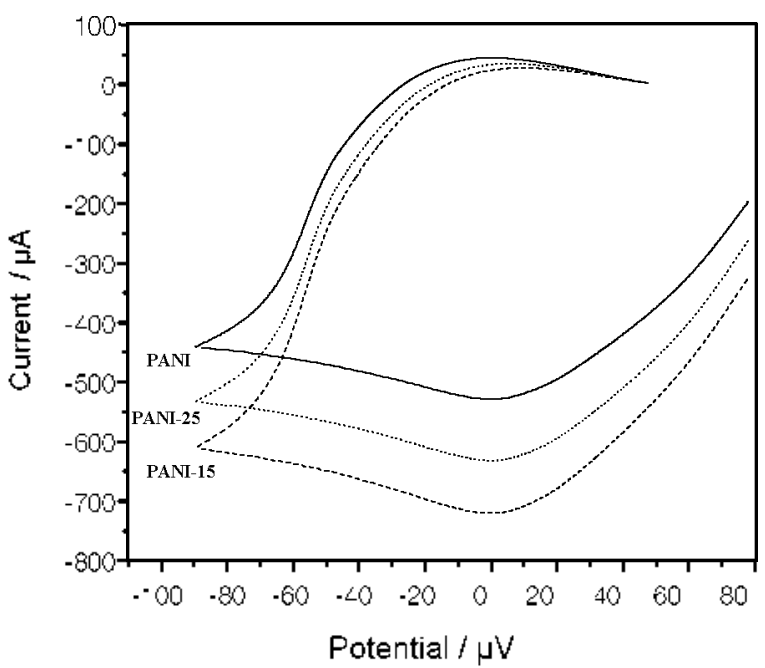

Figure 4. Cyclic voltammograms of PANI, PANI-1 5 and PANI-25 at the scan rate of $10 \mathrm{mV} \mathrm{s}^{-1}$. 
modification in the composite structure of PANI. Continuous stidies are in progress to elucidate the mechanism responsible for the increased electroactivity of PANI-PM composites.

\section{Conclusion}

The PANI-PM composite was successfully synthesized by the chemical polymerization of aniline containing welldissolved PM. The aligned PM chains were encapsulated in the cores of the growing PANI poly mer chains. resulting in the formation of a PANI-PM hybrid material. The high viscosity and complex nature of PM revealed an improvement in physical and chemical properties of PANI such as conductivity and charge carrier mobility: The electrochemical activity of these composites was enhanced with a slight modification in the composite structure of PANI. The improvements made in the various properties of the present $\mathrm{PM}$ are expected to enhance the application potential of the conducting polymer without hampering its chemical properties. The method described here may be useful for developing new applications of these nano-composite films in molecular electronics and other fields. It may be expected that the attractive potentialities offered by this PANI-PM host matrix will be exploited for the development of biosensors based on microorganisms.

Acknowledgments. This work was supported by the 2008 Inje University Research Grant.

\section{References}

1. Gewu, L. U.: Chen, F.; Xufeng, W. U.; Liangti, Q. U.; Zhang, T.; Shi, G. Chm. Sci. Bull 2005, 50(16), 1673.

2. Gill, E: Arousian, A.; Khalil, A: Olga, K. Sensors 2007, 7, 3329

3. Ninel, P. B.: Anna, A. K.; Sergey, V. T.: Larisa, V. K. J. Solid State Electrochem. 2007, 11. 378.

4. Yuvrai, S. N.; Adhyapak, P. V. J. Afacromol. Sci-Potym. Rev: 2002, C $+2(1), 35$

5. Benjannin, I. A.: Richard, W. S.; Linda, S. S. Macromolecules $2004,37(4), 1358$
6. Schultze, J. W: Karabulut, H. Electrochimica Acta 2005. $50(7-8), 1739$

7. Marina, M.: Cátiá, A.: Francesca, S. Solid State lonics 2002. $1+8(3-4), 493$

8. Dhawan, S. K.; Singh, N.; Rodrigues, D. Science and Techlolog' of Advanced Materials 2003, $+(2), 105$.

9. Sarswati, K.: Chandra, R.; Dhawan, S. K. Sensors and Actuators B: Chemical 2001, 75(3), 151 .

10. Shilpa, J.; Sanjay, C.; Samui, A. B.; Krishnamurthy, V. N.; Bhoraskar, S. V. Sensons and Actuators $B$ : Chentical 2003, 96(1-2), 124

11. Franco, D. R. A.; Evandro, G.; Jane, Z. F.; Marco, A. S. R.; Carlos, A. F. J. Mem. Sci. 2004, 234(1-2), 139.

12. Gupta, R. K: Singh, R. A. J. Non-Chystalline Solids 2005 , $351(24-26), 2022$

13. Philip, N. B.; Evelyne, S. Phys. Chent Chent Phys. 2000, 2, 2599.

14. Bartlett, P. N.; Wallace, E. N. K. J. Electroanal. Chem. 2000. $\$ 86(1), 23$

15. Oleg. A. R.: Eugenil, K.: Andreas, F. B.: Itmar, W. J. Am. Chem. Soc. 2002, $12+(22), 6487$.

16. Ronald. E. P.: Roy. D. K.: Tose. P. T. Sensors and Actuators A. Phusical 1998, 6+(1), 77 .

17. Zhao, H.: Liu, H.; Chen, Y.: Xin, X.; Li, J.; Hon, Y.: Zhang, Z:; Zhang, $\mathrm{X}$; Xie, $\mathrm{C}$; Geng, M.; Ding, J Cancer Res. 2006, 66 , 8779

18. Jeong, I. T; Kim, J. H.; Kim, C. K.; Hwang, I ; Lee, K. ificrobiologv $2003,1+9,3265$.

19. Halaas, O: Olsen, W. M:- Veiby, O. P; Lovhaug D; Skjak, B. G.; Vik, R.; Espevik, T. Scond. J. Inmmol. 1997, 46,358.

20. Jahr, T. G.; Ryan, L.; Sundan, A.; Lichenstein, H. S.; Skjak, B. G.; Espevik, T. Infect. Immun. 1997,65,89

21. Liew, C. V.; Chan, L. W. Ching, A. L.: Heng, P. W. hit. J. Pham. 2006, 309, 25

22. Basavaraja, C.; Veeranagouda, Y.: Lee, K.; Pierson, R:; Huh D S. J. Poly Sci.: Pait B: Polv. Phs 2009, 77, 36.

23. Basavaraja, C.; Veeranagolda, Y.: Lee, K.; Pierson, R.; Huh D S. Bull. Kolean Chem. Soc. 2008, 29(12), 2423.

24. Aysegul. G.: Bekir. S.: Muzatfer. T. Stnth. Wet. 2004. 142(1-3). 41

25. Kurt, I. D.; Gudmund, S. B.: Olav, S. Im. J. Biol. Macromolecules $1997,21,47$

26. Dao-jin, G.; Hu-lin, L. J. Solid State Electrochem. 2005, 9, 445.

27. Prakash, R. S. Moter: Chem. Phvs. 2002, 77(1),81.

28. Patil, S. F.; Bedekar, A. G.; Chitra, A. Hater Lett. 1992, 1f(5-6), 307 